\title{
The Norwegian Polio Study 1994: a nation-wide survey of problems in long-standing poliomyelitis
}

Lena Lande Wekre, Johan Kvalvik Stanghelle, Bjørn Lobben and Steinar Øyhaugen

Sunnaas Rehabilitation Hospital, 1450 Nesoddtangen and The National Society of Polio Victims (LFPS), Boks 9217, 0134 Oslo, Norway

\begin{abstract}
'The Norwegian Polio Study 1994' was performed to make a nation-wide survey of the medical and social situation, and of the needs of anterior poliomyelitis (polio). A questionnaire, consisting of 133 questions with sub-questions, was sent to a total of 2392 polio victims, most of them registered in 'The National Society of Polio Victims' in Norway. 1449 persons $(61 \%)$ answered. Sixty-six per cent were between 45 and 64 years of age, $25 \%$ were above 64 years and 9\% were under 45 years. When specifying new health problems, $85 \%$ stated that they had experienced increased weakness in muscles affected by polio, while $58 \%$ had experienced increased weakness in previous non-affected muscles. Other health problems related to polio were fatigue during exercise $(80 \%)$, general fatigue $(57 \%)$, joint pain $(58 \%)$, muscular pain $(58 \%)$ and cold intolerance $(62 \%)$. The participants indicated an increasing need of aids, but $80 \%$ were still independent of help from others and $57 \%$ were still employed, fully or part time. Only $17 \%$ were satisfied with the public health services for polio survivors, while $67 \%$ of those who had undergone comprehensive examination at some central hospital were satisfied. This study indicates an obvious need of building up expertise in multidisciplinary evaluation and treatment of post polio problems in countries where acute polio has been eliminated.
\end{abstract}

Keywords: poliomyelitis; national survey; rehabilitation; health services

\section{Introduction}

During recent years a large number of studies have been presented and have discussed the complexity of new health problems in polio survivors. ${ }^{1-9}$ However, only a few studies have included a larger nation-wide polio population. Most studies have consisted of smaller and selected polio populations at different hospitals, which means that there may be doubts about the size of the need of building up specialized treatment for this group of patients.

This article presents some results from one of the largest nation-wide surveys among polio survivors. 'The Norwegian Polio Study 1994', including a total of 2392 polio victims. Even if the number of polio survivors in Norway probably is two - four times higher, the included group should give representative results of the medical and social situation of these patients.

In addition, we also wanted to find out how the patients evaluated the present health care system, especially when dealing with post polio problems.

\section{Methods}

This study is based on a questionnaire consisting of 133 questions with sub-questions. The questionnaire

Correspondence: LL Wekre was divided into the following sections; gender and age, sociomedical aspects, psychosocial situation, the severity of the poliomyelitis in the different phases (disability, dependency on technical and personal aids), and their relation to the health care system. The questionnaire was prepared by 'The National Society of Polio Victims' (LFPS), in co-operation with several professionals. $^{10}$ However, the questionnaire did not undergo proper validation before being sent out.

In this paper we present the biological facts, the site of paralysis in different phases, the need of personal help, the need of technical aids and the contact with the health care system.

The questionnaire was mailed to a total of 2392 polio victims, 1288 women and 1104 men. Of those were $2064(86 \%)$ members of the LFPS. The remaining 398 were other polio survivors occasionally known. 1449 persons $(61 \%), 980$ women and 459 men, answered the questionnaire, without any collecting letter. Fourteen answers were excluded because of insufficient information.

\section{Statistics}

The results were analyzed, mainly by Feedback Research AS, Oslo, Norway, using the statistic programme SPSS, and with a population of 1444 , 
because the material was adjusted to the normal population in Norway according to gender and population in the different counties.

'The All Possible Pairs test' was used to test the significance. The significance level was $P<0.05$.

\section{Results}

Age and gender

Figure 1 presents the distribution of age and gender of those who answered the questionnaire. Sixty-six per cent of the subjects were between 45 and 64 years of

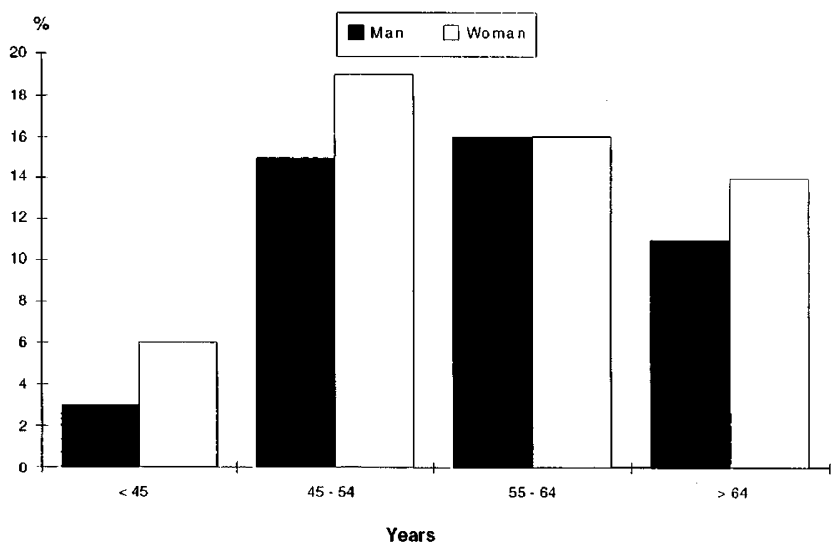

Figure 1 The distribution of age and gender among 1444 responders in the survey age, 25\% were above 64 years and 9\% were under 45 years.

\section{The onset of poliomyelitis}

In an attempt to analyse the representativeness of the study group in relation to survivors of acute poliomyelitis, the number of responders was compared with the number of acute polio cases notified to the medical health authorities each year from 1925 to 1964 (Figure 2). The figure reveals that the responders represent about one tenth of the notified cases, with equal distribution of representation throughout the whole period. The response rate was slightly lower in the Northern part of Norway.

\section{Site of paralysis}

Reported site of paralysis in the different phases is presented in Table 1. Most responders stated that they had experienced new paralysis in the period between the stable phase and today.

New health problems

The frequency of new health problems is given in Table 2. The most frequent complaints were increased weakness in previously affected and unaffected muscles, pain in muscles and joints, considerable fatigue during exercise, considerable fatigue in gen-

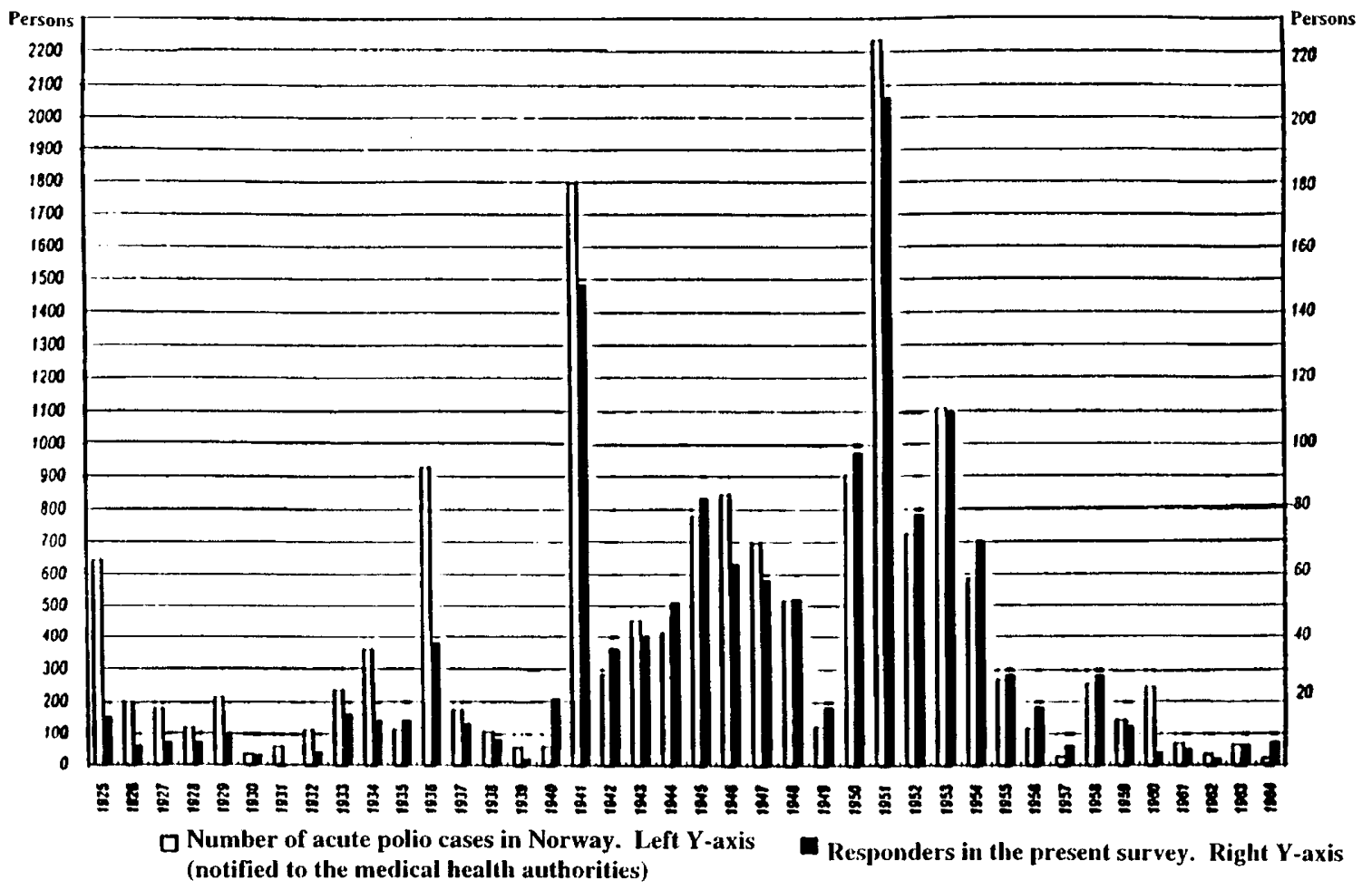

Figure 2 The number of responders in the present survey, compared with the number of acute polio cases notified to the medical health authorities each year from 1925-1964 
Table 1 Reported site of paralysis in the different phases of polio, among 1444 polio survivors. The figures include all who stated some, moderate and total paralysis, given in accurate numbers and in per cent of all responders

\begin{tabular}{lccc}
\hline $\begin{array}{l}\text { Site of } \\
\text { paralysis }\end{array}$ & $\begin{array}{c}\text { Acute } \\
\text { phase }\end{array}$ & $\begin{array}{c}\text { Stable } \\
\text { phase }\end{array}$ & $\begin{array}{c}\text { Situation } \\
\text { today }\end{array}$ \\
\hline One extremity & $346(24)$ & $432(30)$ & $417(29)$ \\
Two extremities & $491(34)$ & $418(29)$ & $403(28)$ \\
Three extremities & $173(12)$ & $158(11)$ & $158(11)$ \\
Four extremities & $375(26)$ & $144(10)$ & $202(14)$ \\
No extremities & $72(5)$ & $302(21)$ & $259(18)$ \\
Back muscles & $792(55)$ & $547(38)$ & $590(41)$ \\
Neck muscles & $475(33)$ & $202(14)$ & a \\
Abdominal muscles & $677(47)$ & $504(35)$ & $547(38)$ \\
Respiratory muscles & $317(22)$ & $173(12)$ & $230(16)$ \\
Throat & $144(10)$ & $58(4)$ & $72(5)$ \\
\hline
\end{tabular}

${ }^{a}$ Not included in the questionnaire

eral, cold intolerance and sleep disturbances. Other frequent reported new problems were numbness, swelling of legs, dyspnoea and concentration problems (Table 2).

\section{Need of aids}

Table 3 presents the use of technical aids, during the stable phase and today, respectively. The frequency of responders who stated a need of technical aids increased, and the frequency of polio survivors who changed from using their technical aids 'sporadically' to 'always', also increased. The number of wheelchair users showed a specially significant rise; 144 subjects had manual wheelchair in their stable phase versus 289 subjects today-and 43 subjects had an electric wheelchair in the stable phase compared with 159 subjects today. The use of respiratory aids and aids for the upper extremities were more than doubled from the stable to the present phase (Table 3 ).

\section{Need of personal assistance}

Most of the responders $(70 \%)$ managed the activities of daily living (ADL) without any personal assistance. However, 19\% needed help for heavy housework and $11 \%$ were totally dependent on personal assistance in ADL. Another 14\% of the responders stated that they actually needed personal assistance at home, but that this was difficult to achieve. The need for personal assistance had increased considerably from the stable to the present phase. As an example, 534 persons stated that they needed assistance carrying on in the stable phase versus 765 persons by the time of the survey.

\section{Employment situation}

In spite of new health problems, $823(57 \%)$ responders were still employed, fully or part time. However, the number of full-time workers had decreased from $65 \%$ in
Table 2 Frequency of new health problems by 1444 polio survivors

\begin{tabular}{lrc}
\hline New health problems & $\mathrm{n}$ & $(\%)$ \\
\hline Weakness & & \\
$\quad$ in previously polio affected muscles & 1227 & $(85)$ \\
in previously polio unaffected muscles & 838 & $(58)$ \\
Pain & & \\
$\quad$ in previously polio affected muscles & 924 & $(64)$ \\
in previously polio unaffected muscles & 794 & $(55)$ \\
Joint pain & 838 & $(58)$ \\
Pain in general & 823 & $(57)$ \\
Fatigue in general & 823 & $(57)$ \\
Fatigue during exercise & 1155 & $(80)$ \\
Numbness & 679 & $(47)$ \\
Cold intolerance & 895 & $(62)$ \\
Concentration problems & 563 & $(39)$ \\
Sleep disturbances & 693 & $(48)$ \\
Swelling of legs and feet & 621 & $(43)$ \\
Dyspnoea & 592 & $(41)$ \\
\hline
\end{tabular}

Table 3 Frequency of users of technical aids, in the stable phase and today, among 1444 responders (per cent)

\begin{tabular}{lcc}
\hline & Stable phase & Situation today \\
\hline Orthopaedic foot wear & $390(27)$ & $418(29)$ \\
1 cane & $260(18)$ & $260(18)$ \\
2 canes & $130(9)$ & $72(5)$ \\
1 crutch & $116(8)$ & $159(11)$ \\
2 crutches & $303(21)$ & $332(23)$ \\
1 short splint & $173(12)$ & $101(7)$ \\
2 short splints & $43(3)$ & $14(1)$ \\
1 long leg caliper & $216(15)$ & $188(13)$ \\
2 long leg calipers & $116(8)$ & $72(5)$ \\
Corset & $317(22)$ & $231(16)$ \\
Wheeled frames (rollator) & $43(3)$ & $58(4)$ \\
Manual wheelchair & $144(10)$ & $289(20)$ \\
Electric wheelchair & $43(3)$ & $159(11)$ \\
Respiratory aids & $14(1)$ & $43(3)$ \\
Aids for upper extremities & $57(4)$ & $115(8)$ \\
\hline
\end{tabular}

the stable phase to $32 \%$ today. Twelve per cent of the participants had the old age pension and $31 \%$ had a different kind of pension from the National Insurance.

\section{Relation to the health care system}

Most of the polio survivors have some sort of contact with the health care system. This survey had questions both about the frequency and the quality of contact. Eighty-three per cent of the participants had their own permanent family doctor. The average time since last contact with this doctor was, however, seven years. Only $14 \%$ of the subjects stated that their medical practitioner was qualified to deal with post polio problems, and $37 \%$ had a desire to change their doctor for their polio problems. Only $17 \%$ stated that they were satisfied with the services from the national health care system. 
Thirty-one per cent of the responders had undergone a multidisciplinary evaluation and treatment due to their post polio problems during the last 5 years, and $67 \%$ of these patients stated that they were satisfied with this evaluation. Another $31 \%$ of the polio survivors wished strongly to have such multidisciplinary evaluation, but for different reasons they had not succeeded in participating in a comprehensive examination and advisory programme.

The results showed a significant geographical difference among the provinces concerning their treatment of post polio problems, and in some provinces there was an obvious lack of medical expertise in this field.

\section{Discussion}

Of the previous epidemiological studies only the Danish post polio survey presents results from a larger polio population, with 3607 participants. $^{2}$ Our survey is a follow-up and supplement to a previous nation-wide survey performed in Norway in 1987, including a total of 1003 polio patients. ${ }^{3}$ The first survey in Norway showed for the first time the complexity of new health problems in polio survivors in our country. However, the results from the first survey is only presented in Norwegian. ${ }^{3}$

The study population of the 'Norwegian Polio Study 1994' was only for registered and other occasionally known polio cases, and it might thus not be representative of the polio survivors in Norway. However, the comparison between our material and all acute polio cases notified by the national health authorities indicated that we had reached a representative part of the Norwegian polio population (Figure 2).

A methodological weakness in this study was the relatively low response rate, probably due to our choice of not sending a collecting letter. The questionnaire was neither validated properly before being sent out. However, the questions were formulated according to comparable studies.

The present survey shows that more than $50 \%$ of polio patients experience new health problems such as weakness in previously affected muscles, considerable fatigue, cold intolerance and pain in muscles and joints (Table 2). These results correspond with the findings of frequent health problems in other published surveys. ${ }^{1-9}$ However, in this study we have not used the term post polio syndrome. Thus we have no figure on the percentage of polio victims who fulfilled the exact criteria of this syndrome. ${ }^{4}$

This study also shows an increased need for technical aids (Table 3), and an increased need for personal assistance, in the polio population. A Norwegian follow-up study of patients with post polio syndrome ${ }^{11}$ also indicates that the problems of these patients increase in spite of considerable intervention. However, many patients improved their psychological health due to the intervention, even if their physical health was reported to be worse ${ }^{12}$ indicating that their ability to cope had improved.

Only $17 \%$ of the responders were satisfied with the health care system. According to our experience with polio patients who attend our rehabilitation hospital, many polio patients feel that they are misunderstood, and many of them experience that the health care system has little knowledge of post polio problems. Thus, many of the polio survivors have to use much extra energy before they obtain the support and treatment that is obviously required.

The results of this study also showed a significant geographical difference concerning the treatment of post polio problems among the provinces in Norway. Those patients who had undergone a multidisciplinary evaluation primarily, lived close to the central hospitals which already had special services for polio patients. The majority of these patients were very satisfied with these services. Thus, the results indicate a need to build up new expertise on post polio problems in all of the provinces.

\section{Conclusion}

This nation-wide survey among 2392 polio survivors shows that more than $50 \%$ of polio survivors in Norway experience new health problems related to their polio, an increased need of technical aids, increased need of personal assistance, and an increased need of multidisciplinary health services. The results also indicate an obvious lack of medical expertise for those with post polio problems. It is therefore important to build up expertise in the multidisciplinary evaluation and treatment for polio victims in all of our provinces, and also in other countries where acute polio is eliminated.

\section{References}

1 Halstead LS, Grimby G, Red. Post-polio syndrome. Philadelphia: Hanley-Belfus, 1995.

2 Lønneberg F. Late onset Polio sequelae in Denmark. Results of a nationwide national survey of 3607 survivors. Scand J Rehabil Med 1993; suppl 28.

3 Norges Handicapforbund: Previous polio patients, how are they today? Oslo 1988. (Norwegian).

4 Halstead LS, Rossi CD. New problems in old polio patients: Results of survey of 539 polio survivors. Orthopedics 1985; 8: $845-850$.

5 Halstead LS, Wiechers DO, Rossi CD. Results of a survey of 201 Polio Survivors. Southern Med J 1985; 78: 1281-1287.

6 Grimby G, Einarsson G, Hedberg M, Aniansson A. Muscle adaptive changes in postpolio subjects. Scand J Rehab Med 1989; 21: $19-26$.

7 Perry J, Barnes BS, Gronley JK. The postpolio syndrome. An overuse phenomen. Clin Ortop and Rel Res 1988; 233: 145-162.

8 Diard C, Ravaud JF, Held JP. French survey of postpolio sequelae. Risk factors study and medical social outcome. Am J Phys Med Rehabil 1994; 73: 264-267.

9 Westbrook MT. A survey of post-poliomyelitis sequelae: manifestations, effects on people's lives and responses to treatment. Australian Physiotherapy 1991; 37: 2. 
Post poliomyelitis problems

LL Wekre et al

10 Bjørn Lobben, Steinar Øyhaugen. 'The Norwegian Polio Study 1994'. Oslo; 'The National Society of Polio Victims' (LFPS) 1995.

11 Stanghelle JK, Festvåg LV. A five year follow-up of patients with post-polio syndrome. Spinal Cord 1997; 35: 503-508.
12 Schanke AK. Psychological distress, social support and coping behavior among polio survivors. A 5 year follow-up on 63 polio patients. Disability and Rehabil 1997; 19: $108-116$ 